BNL - 67709

CAP-288-NuFact-00C

Informal Report

\title{
Power Compression of Low-Frequency RF by Means
} of a Thyratron

\author{
Yongxiang Zhao
}

Brookhanen National Laboratory, Upton, New York

September 2000

Pres. at " $2^{\text {nd }}$ Intn'1. Workshop on "Neutrino Factories Based on Muon Storage Rings (NuFact'00), Monterey, CA, May 22-26, 2000. Not submitted to Proceedings. 


\title{
POWER COMPRESSION OF LOW-FREQUENCY RF BY MEANS OF A THYRATRON
}

\author{
Yongxiang Zhao \\ Fhysics Department, Bldg. 901-A \\ Brookhaven National Laboratory, Upton, NY 11973
}

\begin{abstract}
To generate brief pulses of RF of low frequency and very high power, the author proposes a novel system of power compression. A tetrode of modest capacity fills a storage RF cavity of very high $\mathrm{Q}$. A switch then diverts the energy into the load cavity. To switch the power, a thyratron should be suitable for frequencies up to perhaps $\sim 200 \mathrm{MHz}$. Some thyratrons are capable of a closed-circuit current of $\sim 10 \mathrm{kA}$ and an open-circuit voltage of $\sim 100 \mathrm{kV}$. With a commercial thyratron the system may deliver a pulse of power of tens even hundreds of megawatts. Presented here are the principles of operation of such a system, a schematic engineering layout and a proposed circuit diagram, including the firing circuit. Theoretically, there is no limitation on the power compression ratio.
\end{abstract}

\section{INTRODUCTION}

The muon collider project requires RF accelerating cavities within a frequency range that is many times lower than that typical of most accelerator facilities: only 30 $\mathrm{MHz}$ to $200 \mathrm{MHz}$. This is because the emittance of the muon beam is very large, especially early in the acceleration process, during the stages of phase rotation and cooling. A severe engineering challenge is to develop the power supplies to drive these low-frequency cavities to the very high electric field gradient required for rapid acceleration. Although at S-band or X-band frequencies klystrons can deliver tens of megawatts, no existing tube can provide more than a few $\mathrm{MW}$ at these low frequencies.

Among the candidate devices that engineers have been investigated are the IOT [Inductive Output Tube] ${ }^{1}$, tetrode ${ }^{2,4}$, inverted tetrode ${ }^{3}$, hobetron ${ }^{5}$, MIG [Magnetron Injection Gun] gun triode ${ }^{6}$, and multi-beam klystron. None has proved satisfactory, because of excessive cost for R \& D and/or technical uncertainties.

A technique applied in many accelerators is that of power compression, which enhances the peak power. Among the many linear compression schemes are SLED [SLAC energy doubler] $]^{7}, \operatorname{SLED}-\Pi^{8}, \mathrm{BPC}^{9}$ (binary power compression), $\operatorname{DLDS}^{10}$ (delay line distribution system) and $\mathrm{VPM}^{11^{*}}$. The SLED method has been the most successful. In the S-band SLED at the Stanford Linear Accelerator Center [SLAC] two equal storage cavities, employed and operated in the $\mathrm{TE}_{015}$ mode, enhance the $\mathrm{Q}$ factor. However, at low frequency the scheme requires cavities that are inconveniently large. Likewise, the other schemes listed above are impractical at low frequencies.

\footnotetext{
*VPM ( VLEEP power multiplier), VLEEP is a collider project in Budker Institute of Nuclear Physics, Novosibirsk., Russia
} 
An alternative to linear compression employs a storage cavity and a switch ${ }^{12}$. A low-level source gradually charges a storage cavity and then discharges it rapidly by opening a high-intensity electron beam switch. The technique thereby can deliver a peak output power that is much larger than the drive power. In principle there is no obvious limitation to the factor of power compression. However, the electron beam switch greatly complicates the system and thus restricts its application.

The key component is the switch. A switch operating at low frequency should be easier to develop than one for high frequency. Frequencies in the tens of $\mathrm{MHz}$ range, as needed by the muon collider, are close to video frequencies; might it be possible to use a thyratron for this frequency range? If the answer is yes, the storage cavity-switch scheme might be feasible and open a door to a new realm of high peak power.

To make this idea convincing, one must design an engineering layout in which the construction and all parameters are practical. This note attempts to accomplish this.

\section{THE PRINCIPLE}

Fig. 1 shows the principle of the compression scheme of storage cavity and switch. To charge the storage cavity, one might employ a cw tetrode. Its output is connected to a half-wavelength cable with a switch at the other end and a load at the midpoint. When the switch is open, as shown in Fig.1 (a), the load branch is at the node of the voltage. Therefore no power goes to the load, and the storage cavity sees an open circuit. When the switch is closed, Fig. 1 (b), the right quarter-wavelength cable acts as an open circuit. The storage cavity then connects directly to the load (e.g., the accelerator cavity), to which the energy drains.

Since the charging time is much longer than the discharging time, the output peak power can be many times larger than the drive power. This accomplishes the power compression

As shown in the Figure, the switch should hold off a voltage $V$ without breakdown when the switch is open, and should carry a current $I=V / Z_{0}$ when its status is closed. The power $P=V I$ is exactly the output peak power, if the load is matched. This suggests that the power handling capability of the switch should be of the same order as the output power. It may be different, if the load is not matched.

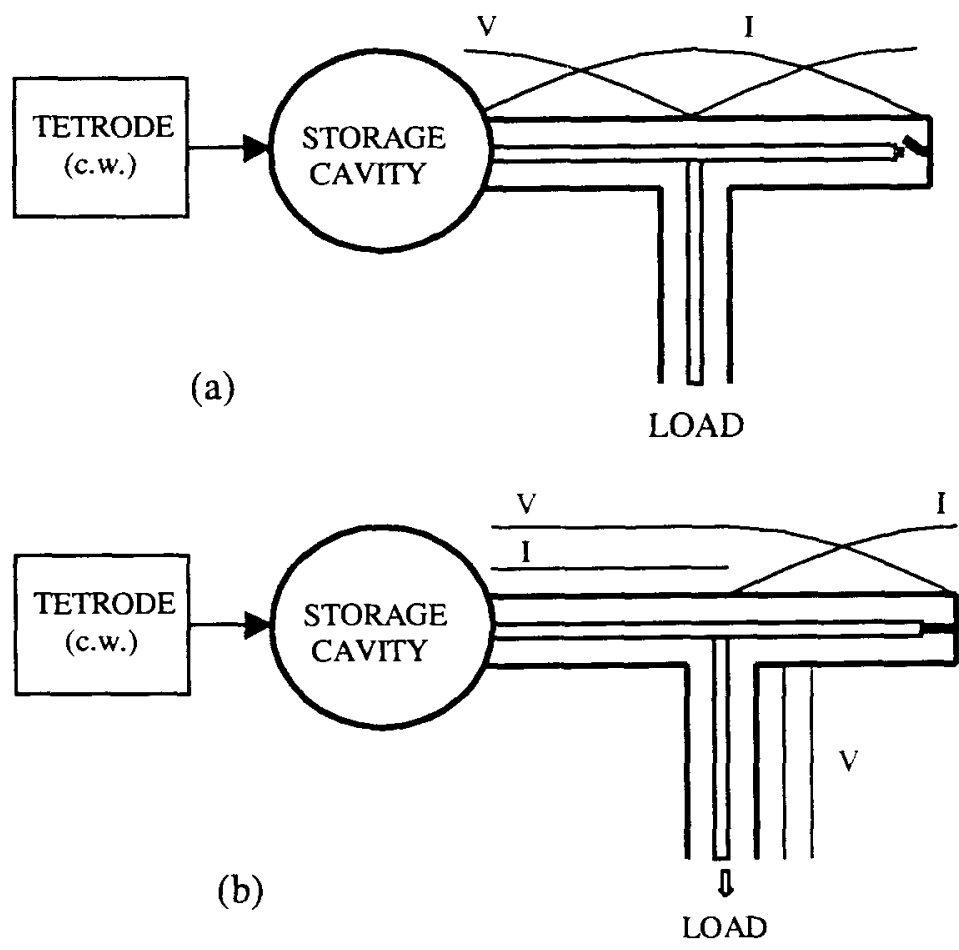

Fig.1 Principal setup of storage cavity - switch compression 
What device can serve as this RF switch? This question has troubled decades of Sand X-band accelerators. A PIN diode is good, but only for moderate power, with the present state of the art. A high-intensity beam switch worked, but proved to be too complex. A TR [transmit-receive] tube used in radar duplex likely would not work, because of poor controllability and inadequate power, to our knowledge.

For our particular case of low frequency, will a thyratron work as an RF switch? Nowadays, commercial thyratrons can control $\sim 100 \mathrm{kV}, \sim 10 \mathrm{kA}$. Such a thyratron can control hundreds of megawatts. If thyratrons can serve as RF switches, the potential for huge peak power may become very attractive.

Two questions arise. First: Will a thyratron behave as an open circuit before firing? The inter-electrode capacitance of a thyratron is in the order of 15 to $20 \mathrm{pF}$. At frequencies that are too high, the thyratron will transmit, not block, the wave. Even at low frequencies, this capacitance will destroy the perfection of the thyratron's open status. One can regard the thyratron capacitance as a piece of stub with an open end. By shortening the length of the cable, one should be able to make the scheme work.

Second: Will the RF current pass through the thyratron? As is well known, a thyratron can pass only positive current, i.e. that passing from the anode to the cathode. However, if one supplies a bias current so as to eliminate the negative current, the condition can be satisfied, as illustrated in Fig.2 (right bottom).

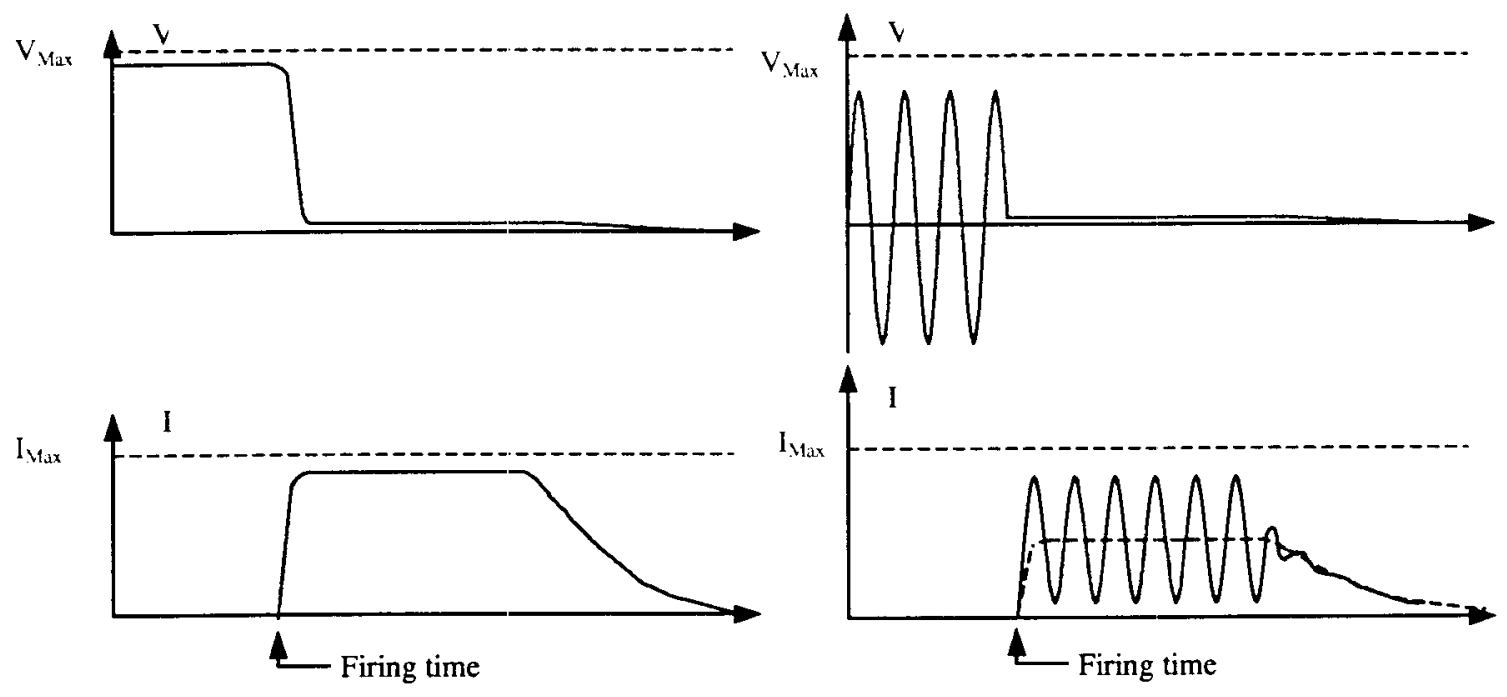

Fig. 2 The anode voltage and current of a thyratron, left-video pulse case, right-RF case

Both the voltage and the current have upper limits specified by the manufacturer. It is necessary that operation be always within these limits. Because a bias current (dashed line in Figure) is superimposed on the RF current, so that the instantaneous current always is greater than zero, the maximum RF current amplitude should be less than half of the allowable upper limit, $I_{\max }$. Whether the specified limits are applicable in the case of RF remains to be studied. 
Also, one should note that, once a thyratron is fired it can not be reopened until all the ions have recombined. This means that the energy inside the storage cavity should be completely discharged before the next charging cycle. This is not a problem in our case, because we are concerned with a duty cycle that is very low. There will be enough time for ion recombination before recharging.

\section{ENGINEERING LAYOUT}

To realize the above idea, one must answer two additional questions. The first is, how to control the firing time? The second is, how to supply the bias current?

Fig. 3 shows a proposed construction. The thyratron is mounted at the end of a 12 inch rigid cable, with the screen grid connected to the external conductor and the anode to the internal conductor. The cable, including both external and internal conductors, must be grounded. The anode should not be grounded, because the anode and the screen grid are at different potentials. To solve this problem, one employs a choke structure that transmits the radio frequency while insulating the video pulse. Of course, one may instead employ a capacitor for ac transmission and $\mathrm{dc}$ isolation. Which technique is better depends on engineering considerations.

The RF field is confined to the inside of the cable. Within the thyratron it is restricted mainly to the space between the anode and the screen grid (or gradient grid in some thyratrons). It does not affect the firing, as long as the RF voltage is within the breakdown limit. The trigger grid is outside the RF field and will control the firing time independently.

Fig.4 shows the schematic circuit. Note that it is different from a normal pulse modulator. There is no $\mathrm{dc}$ high voltage applied to the anode of the thyratron. Instead, one applies a pulse of high voltage by means of a special trigger synchronized with the

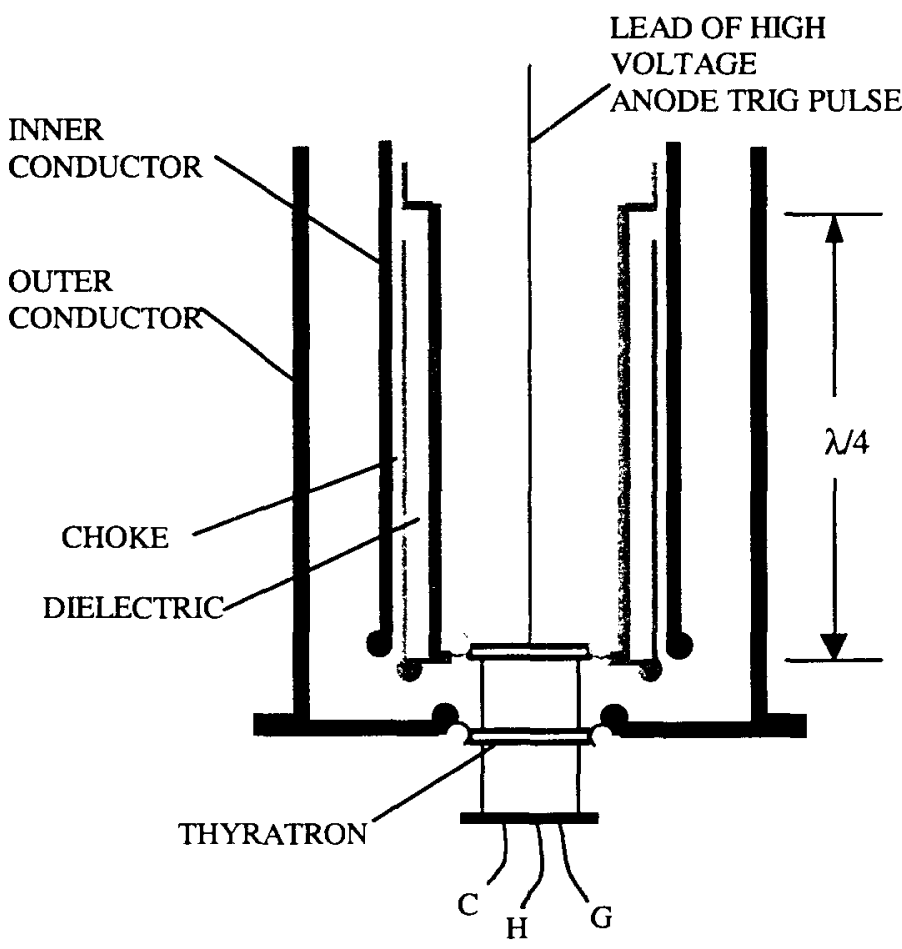

Fig. 3 The assembly of the cable and thyratron

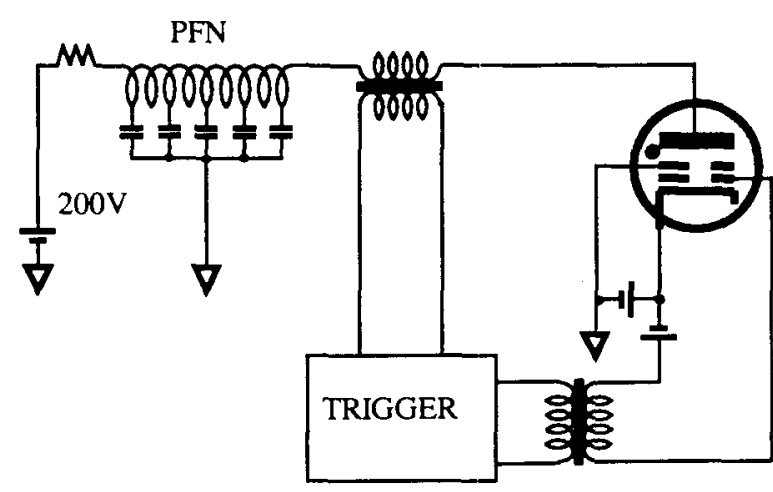

Fig. 4 The trigger and bias assembly 
trigger pulse to the first grid. The pulse on the anode enables the thyratron to fire.

Once the tube is conducting, the PFN (pulse forming network) will supply a long pulse of current to sustain the plasma inside the thyratron. This current is the bias current mentioned above. The pulse length has to cover completely the RF discharging time. However, the voltage of the PFN is only $200 \mathrm{~V}$ or so, the voltage drop of the thyratron during its conducting period. (The exact value depends on the tube used.) The PFN is not high-energy storage, and therefore is not costly.

Thus the thyratron withstands both $\mathrm{RF}$ and video voltages, which come from different sources, nearly independently of each other.

\section{NUMERICAL ANALYSES}

The feasibility of the scheme depends on all parameters being in reasonable ranges. Two parameters are of most concern: (1) How great a $Q$ value is required for the storage cavity? (2) How great a compression ratio can one obtain?

First, we need to know the total energy consumed by the load. If the accelerator is a cavity with known maximum stored energy $W_{a c}$, the minimum energy in the storage must be at least $W_{a c}$. This will be an underestimate, because it would require all of the energy to be transferred from one cavity to another with $100 \%$ efficiency ${ }^{*}$. Let's estimate the minimum energy in another way. Suppose that the intrinsic $Q$ factor of the accelerator is $Q_{a c}$. Then the power required is:

$$
P_{a c}=\frac{\omega W_{a c}}{Q_{a c}}
$$

The filling time is:

$$
\tau=\frac{Q_{L}}{\pi f}=\frac{Q_{a c}}{1+\beta} \frac{2}{\omega}=\frac{Q_{a c}}{\omega} \quad(\text { when } \beta=1) .
$$

Normally, the charging time would be about three times the filling time, or $\sim 3 \tau$. Then the total energy absorbed by the accelerator is $W_{L} \approx 3 \tau P_{a c}=3 W_{a c}$. The stored energy should, of course, be larger: $W_{s}>3 W_{a c}$.

For a traveling wave structure with matched coupling, this estimate still is valid.

The drive power to charge the storage cavity is:

$$
P_{d r}=\frac{\omega W_{s}}{Q_{s}}>3 \cdot \frac{\omega W_{a c}}{Q_{s}}=3 \cdot \frac{Q_{a c}}{Q_{s}} P_{a c} .
$$

$Q_{s}$ is the system $Q$, which includes the internal and external loss when the switch is open. The power gain (or compression ratio) is:

\footnotetext{
* Theoretically, for a very high $Q$ system involving two resonators, energy swings between them with high efficiency is possible, providing breakdown is not a problem.
} 


$$
G=\frac{P_{a c}}{P_{d r}}<\frac{1}{3} \frac{Q_{s}}{Q_{a c}} .
$$

One can rewrite this inequality as $Q_{s}>3 G Q_{a c}$. If one wants high gain, the $Q$ value of the storage must be correspondingly high. For example, if one desires a compression ratio of $G=20$, assuming the accelerator has a $Q$ of $Q_{a c}=10 \mathrm{k}$, then the storage cavity must have a minimum $Q$ of $Q_{\text {smin }}=60 Q_{a c}=600 \mathrm{k}$.

A copper cavity can achieve this value, but only if it operates in the TE01 mode. Unfortunately, a cavity in this mode must have a diameter and height that are comparable with the wavelength. For the low frequencies of interest, e.g. $30 \mathrm{MHz}$, for which the wavelength is 10 meters, the cavity size is much too big to be practicable. Therefore, one needs a superconducting cavity. However, we do not require a cavity of the highest quality, for which $Q$ may be $10^{9}$ to $10^{10}$. A $Q$ of $10^{8}$ or so should be adequate.

The power gain, or the compression ratio, is the ratio of the output power to the tetrode power, which drives the storage cavity. The latter power depends on how high a loaded $Q$ one can achieve when the switch is open $\left(Q=Q_{e o}\right)$. The higher the $Q_{e o}$, the less drive power one needs. The output power depends on how low a loaded $Q$ one can achieve when the switch is closed $\left(Q=Q_{e c}\right)$. The lower the $Q_{e c}$, the faster is the release of energy. Therefore, one needs to calculate the ratio $Q_{e d} / Q_{e c}$, which is a measure of the quality of the switch system. With a superconducting cavity the internal $Q$ is much higher than the external one, the above ratio takes only the external loss into account. Thus one can calculate the ratio of the impedance from the outlet of the storage cavity, as shown in Fig.5.

$$
\frac{Q_{e o}}{Q_{e c}}=\frac{Z_{e o}}{Z_{e c}}
$$

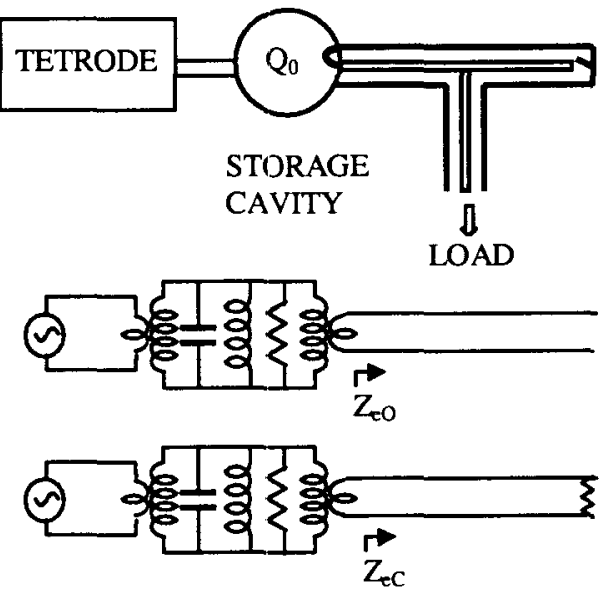

Fig. 5 The equivalent circuit

where $Z_{e o}$ and $Z_{e c}$ are, respectively, the output impedances of the storage cavity when the switch is open and closed (see Fig.5).

To the first order of approximation, we omit the loss of the switch itself. Since the voltage drop of a thyratron in conducting status is nearly constant, independent of the RF current, its RF resistance is very small. Therefore, we need take into account only the loss of the transmission line. The impedance of a transmission line is:

$$
Z_{e}=Z_{0} \frac{Z_{l}+j Z_{0} \tan k l}{Z_{0}+j Z_{l} \tan k l}
$$

$Z_{0}$ is the characteristic impedance of the transmission line; $Z_{l}$ is the load impedance at the termination; and $l$ is the length and $k$ the propagation constant. In a lossy line $k$ is complex: $k=k_{1}+j k_{2}$, where $k_{I}$ is the phase constant and $k_{2}$ the decay constant.

With the switch in open status, $Z_{l}=\infty$; then $Z_{e o}=Z_{0} / j \tan k l$. 
Note that the output load is joined at the voltage node; it absorbs little power, which we have ignored here. As mentioned above, $k_{l} l=\lambda 2$, and normally we have $k_{2}<<1$, or $\tan k l=\tan \left(k_{1} l+j k_{2} l\right)=-j \tanh \left(k_{2} l\right) \approx-j k_{2} l$.

Therefore:

$$
Z_{e o} \approx Z_{0} / k_{2} l \text {. }
$$

For the switch in its closed status, $Z_{l}=0$. In this case the right half of the transmission line is a quarter-wavelength short stub, which functions as an open end. Strictly speaking, it is not fully open, because the line and the switch are lossy and will absorb some power. However, for the time being we consider the accelerator to be a matched load. Then the loss in the switch branch can be ignored in comparison with the parallel matched branch, which absorbs the majority of power. Consequently, the outlet of the storage cavity sees a simple matched load:

$$
Z_{e c}=Z_{0}
$$

Finally, we get:

$$
\frac{Q_{e o}}{Q_{e c}}=\frac{1}{k_{2} l} .
$$

Assume a 12 ", $50 \Omega$ rigid copper cable, $f=30 \mathrm{MHz}, l=\lambda \Omega$, skin depth $\delta=12 \mu \mathrm{m}$. Eventually one can find:

$$
\frac{Q_{e o}}{Q_{e c}}=\frac{1}{k_{2} l}=4100 .
$$

The coupling coefficient of the storage cavity, $\beta=Q_{d} / Q_{e}$, is inversely proportional to $Q_{e}$. The above ratio is also the ratio of coupling coefficients, i.e. $\beta_{e c} / \beta_{e o}=Q_{e d} / Q_{e c}=4100$.

The coupling is not decided yet and remains arbitrary. But, obviously, we hope that it is under-coupled during the charging period, so as to have minimal leakage from storage. On the other hand, it should be strongly over-coupled during discharge, so as to discharge fast. Since its ratio is around 4000 , we assume $\beta_{e o}=0.5, \beta_{e c}=2000$.

With this value of $\beta_{e o}$, the drive power is:

$$
P_{d r}=\frac{\omega W_{s}}{Q_{s}}\left(1+\beta_{e o}\right),
$$

and the output power is: $\quad P_{\text {out }}=\frac{\omega W_{s}}{Q_{e c}}$.

Then the power gain is: $\quad G=\frac{P_{\text {out }}}{P_{d r}}=\frac{\beta_{e c}}{1+\beta_{e o}}=\frac{2000}{1+0.5}=1330$.

This number is much larger than the minimum desired value of 20 . Though the imperfection of the switch will reduce this value, it still is encouraging that the theoretical gain can be so high, $>1000$. 


\section{ACKNOWLEDGEMENTS}

This work was supported by the US DOE under Contract No. DE-AC0298CH10886. Thanks also to all the members in CAP group and muon collider community who are interested and encouraged this work. My special gratitude to R. Weggel who review my draft and made significant revise.

\section{References}

[1] D. Wilcox, (EEV) "Preliminary concepts for very high power IOTs", Muon Collider Collaboration Meeting, May 20-26, 1999, St. Croix, US Virgin Islands.

[2] V. Mason, (Eimac) "Prospects for a high-power tetrode tube", ibid.

[3] Y. Zhao, "A high power inverted tetrode tube", ibid.

[4] G. Cleric, (Thomson) "RF power sources in the 20/200 MHz range", Muon Collider Experimental RF System Meeting”, LBNL, Feb. 18-19, 1999.

[5] R. True, (Linton) "Introduction to the hobetron", ibid.

[6] R. Palmer, "MIG gun triode", ibid.

[7] Z. D. Farkas, "SLED: A method of doubling SLAC's energy", Proc. of 9th Int. Conf. on High Energy Accelerators, p.576, 1976.

[8] P. B. Wilson, et al. "SLED-II: A new method of RF pulse compression", Linear Accelerator Conference, Albuquerque, NM, Sept 1990; SLAC-PUB-5330.

[9] Z. D. Farkas, "Binary peak power multiplier and its application to linear accelerator design”, IEEE Trans. MTT-34, pp.1036-1043, 1986.

[10] H. Mizuno, 'X-band RF system for JLC", Workshop of RF96 - Pulse RF source for linear collider, Shonan Village Center, Japan, Apr. 8-12, 1996.

[11] V. Balakin, I. Syrachev "Status of VLEEP power multiplier", Proc. of 3rd Int. Workshop on next generation linear collider" Sep, 1991, Protivino, Russia; "Open cavity RF pulse compression system" Workshop of RF93 - Pulse RF source for linear collider.

[12] D. L. Birx et al. "Microwave energy compression using a high-intensity electron beam switch", J. Appl. Phys. 51(7), p. 3629, July 1980. 